\title{
A new and simplified functional tendon transfer for a dropped hallux
}

\author{
Sukhbir Singh, Tejbir Singh ${ }^{1}$ \\ Department of Burns and Plastic Surgery, Lok Nayak Hospital, New Delhi, ${ }^{1}$ Kamal Hospital, Kaushambhi, India
}

Address for correspondence: Dr. Sukhbir Singh, S-212, Greater Kailash Part-2 New Delhi-110 048, India. E-mail: sukhi_1@yahoo.com

\section{ABSTRACT}

A case of fracture of the right tibia proximal $1 / 3$ rd extending up to diaphysis after a road traffic accident along with a dropped hallux is being reported. The extensor digitorum longus (EDL) was intact. Patient was taken up for surgery for the fracture tibia with conservative management for his dropped hallux for initial six weeks. After six weeks when no improvement was seen surgical intervention was planned for the dropped hallux. The subsequent management of this patient is being described.

\section{KEY WORDS}

Dropped hallux; extensor digitorum longus; extensor hallucis longus; extensor hallucis brevis

\section{INTRODUCTION}

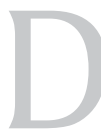

ropped Hallux is not a common entity and we present a simplified approach for its correction. We transferred the first $\left(1^{\text {st }}\right)$ slip of EDL and also the extensor hallucis brevis (EHB) to extensor hallucis longus (EHL), thus achieving the final postoperative result as depicted in the figures and videos. Patient subsequently developed good range of motion postoperatively with 0-15 degrees of extension and 0-15 degrees of flexion.

\section{CASE REPORT}

A 55-year-old male sustained a closed fracture of the right tibia following a road traffic accident. On admission, his vitals were stable. Circulation of the extremity was compromised, so immediate fasciotomy was done to relieve the excessive limb swelling. His X-rays were done revealing a Schatzker ${ }^{[1]}$ type 6 injury along with dropped right hallux. Patient was taken up for surgery for his fracture tibia and plating was done along with split skin grafting of the fasciotomy wound and primary closure of his other wounds. The hallux was treated symptomatically by splinting and giving a slab with foot in dorsiflexion. Patient recovered completely except for the dropped hallux. Conservative management of the same was continued for six weeks after which surgical intervention was planned.

\section{Procedure}

Surgical exposure was done exposing the extensor hallucis longus (EHL), extensor hallucis brevis (EHB) and the $1^{\text {st }}$ slip of the extensor digitorum longus (EDL) tendon was exposed. Our approach has been explained with the help of a line diagram [Figure 1]. We anchored the EHL tendon to EHB tendon and instead of completely detaching the $1^{\text {st }}$ slip of EDL, we longitudinally split it starting $1.5 \mathrm{~cm}$ proximal to its insertion as shown in the line diagram [Figure 1] and anchored it to the EHL .

\section{Postoperative protocol}

Initially splintage was continued for six weeks followed by physiotherapy, which was combined with night splintage for another two weeks. The physiotherapy 


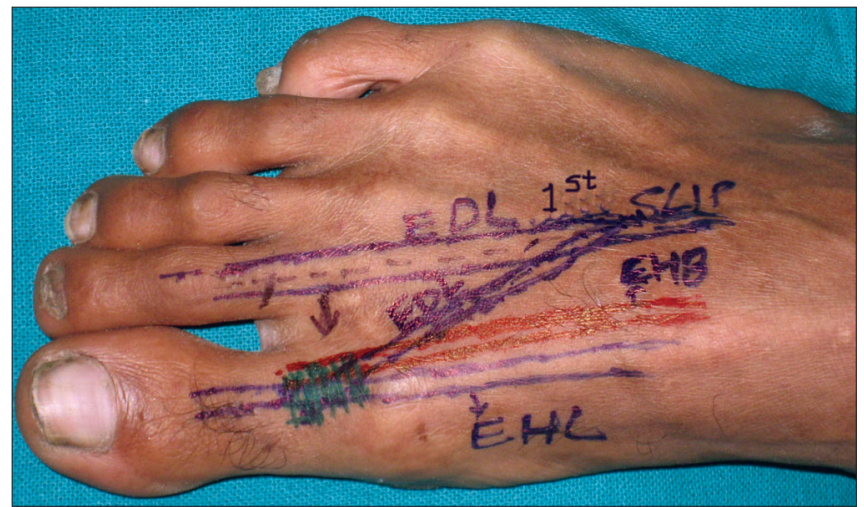

Figure 1: Line diagram showing dynamics of transfer

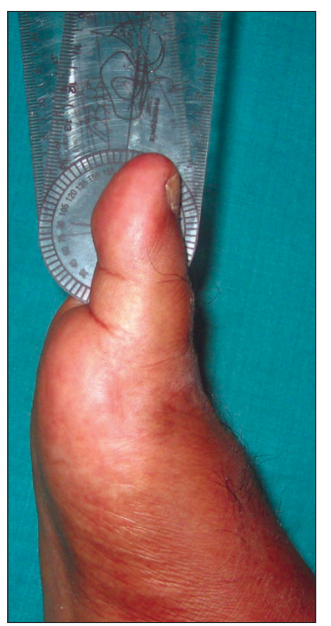

Figure 3: Great toe in active extension and measurement with goniometer

done was active under supervision initially and patient was subsequently trained to do the same all by himself. The postoperative recovery was satisfactory with a small scar line [Figure 2], which healed uneventfully. The range of motion was measured using goniometer. The range of motion postoperatively is 15 degrees of extension and 15 degrees of flexion. The ranges of motion of both sides have been compared in Table 1. Active extension of the hallux is shown in Figure 3. Both active flexion and extension movements have been depicted in the Videos 1 and 2.

\section{DISCUSSION}

Damage to either the EHL muscle belly or the motor nerve to EHL are uncommon causes of the dropped hallux. ${ }^{[2]}$ This problem can occur after injury, high tibial osteotomy or intramedullary nailing of a fractured tibia. ${ }^{[1]}$ Very little literature is present on the treatment aspects of the same. Similar technique which we have described could not be identified after extensive search of literature. The cause of the dropped hallux in our case was most

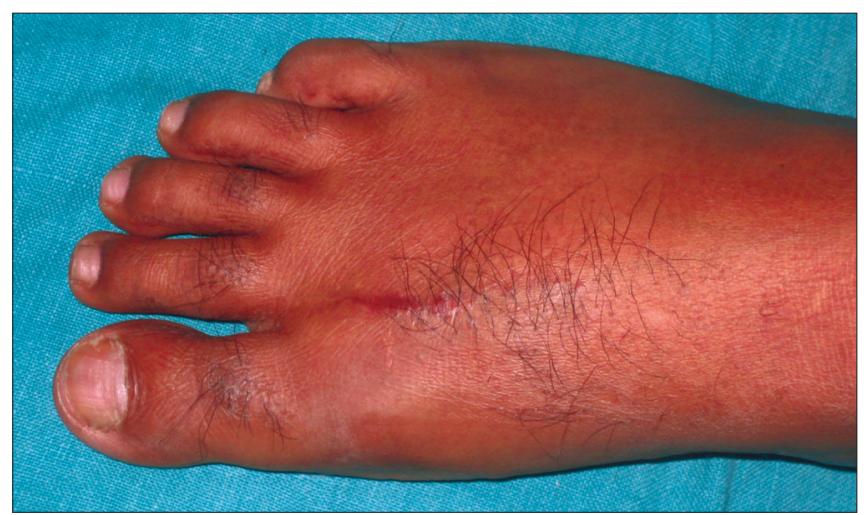

Figure 2: Postop result with healed short scar

Table 1: Comparison of range of motion at first metatarsophalangeal joint (MTP Joint) b/w normal (left foot) and operated side (right foot)

\begin{tabular}{lcc}
$\begin{array}{l}\text { Range of motion at first } \\
\text { metatarsophalangeal joint } \\
\text { (MTP Joint) }\end{array}$ & $\begin{array}{c}\text { Normal left } \\
\text { foot }\end{array}$ & $\begin{array}{c}\text { Operated } \\
\text { right foot }\end{array}$ \\
\hline Flexion at first MTP joint & 0 to 15 & 0 to 15 \\
degrees & degrees \\
Extension at first MTP joint & $\begin{array}{c}0 \text { to } 18 \\
\text { degrees }\end{array}$ & $\begin{array}{c}0 \text { to } 15 \\
\text { degrees }\end{array}$ \\
\hline
\end{tabular}

probably due to detachment of the EHL tendon from its origin following the trauma. There was no sensory loss ruling out any injury to the deep peroneal nerve.

\section{Dynamics of function}

Our transfer follows the general principles of tendon transfer. Firstly, we ensured before the transfer that the joints were supple and there was no soft tissue scarring in the area of transfer. Secondly, we have used both the extensors (i.e., EHB and $1^{\text {st }}$ slip of EDL), which are agonists for the extensor function we intended to achieve by the transfer. Thirdly, the transfer tendons were lying in a straight path as required for effective functioning of the tendon transfer. Lastly, we have not compromised any function due to the transfer since we have split the $1^{\text {st }}$ slip of EDL longitudinally and transferred it to the EHL thereby retaining the active $2^{\text {nd }}$ toe extension. Postoperatively the range of motion was also satisfactory with only 3 degrees of extensor lag as shown in Table 1.

Tendon transfers have been described for clawing of great toe viz. the Jones ${ }^{[3]}$ tendon transfer and the Hibbs ${ }^{[4]}$ procedure, however, transfers for dropped hallux posttrauma have not been clearly mentioned or detailed in literature. We have presented a simplified approach which is quick with less tedious dissection, and produces good results and patient satisfaction. 


\section{See video on www.ijps.org}

\section{REFERENCES}

1. Schatzker classification for tibial fractures. Campbell's Ope Ortho 2003;3:2783-4.

2. Smith AM, Modarai B, Davies M, Birch R. Extensor hallucis longus to extensor digitorum communis tendon transfer: $A$ treatment for extensor Hallucis Longus dysfunction. J Bone Joint Surg Br 2003;85:250.
3. Transfer J. Transfer of EHL for claw toe deformity. Campbell's Ope Ortho 2003;3:1380.

4. Transfer of Extensor tendons to Middle Cuneiform for claw toe deformity. Hibbs procedure. Campbell's Oper Ortho 2003;3:1380.

Source of Support: Nil, Conflict of Interest: None declared. 